\title{
ANÁLISIS TEÓRICO-EXPERIMENTAL DEL COLECTOR SOLAR CILÍNDRICO PARABÓLICO AUTOMATIZADO CON ESPEJOS REFLECTANTES
}

\author{
THEORETICAL AND EXPERIMENTAL ANALYSIS \\ OF AUTOMATIC CYLINDRICAL PARABOLIC SOLAR \\ COLLECTOR WITH REFLECTIVE MIRRORS
}

\author{
Anthony Pinedo Araujo ${ }^{1}$ y David Asmat Campos ${ }^{2}$ \\ ${ }^{1}$ Universidad Privada del Norte, sede Trujillo, Av. El Ejército 920, Urb. El Molino, Trujillo \\ ${ }^{2}$ Universidad Nacional de Trujillo, Av. Juan Pablo II S/N, Ciudad Universitaria, Trujillo
}

\section{RESUMEN}

En la presente investigación se presenta un análisis de los colectores solares cilíndricos parabólicos (CCP), los cuales fueron mejorados a través de la incorporación de espejos reflectantes, aumentando así la eficiencia de los mismos. De igual manera, se añadió un sistema de seguimiento de luz basado en fotosensores para aprovechar al máximo la irradiación solar. Como primera aproximación, se asumió que el colector solar funciona como un cuerpo negro perfecto. Luego, se propone un modelo más real, en donde se toman en cuenta las pérdidas de calor, la disipación de los rayos solares, las propiedades ópticas de los materiales utilizados, etc. En la parte experimental, se armó todo el sistema, comparándolo con los sistemas tradicionales de los CCP, obteniendo una notable mejora en la temperatura alcanzada por el tubo receptor en un menor tiempo de exposición. Asimismo, se presentan las aplicaciones de este tipo de colectores, que van desde sistemas domésticos de calefacción, mejoramiento de producción anaeróbica de fermentación, hasta centrales termosolares para la generación de energía eléctrica. En la parte final, se presentan propuestas para la mejora de este sistema en función al estudio teórico realizado.

Descriptores: colector cilíndrico parabólico, seguimiento de luz, energía solar, espejos reflectantes

\begin{abstract}
In this research we show an analysis of cylindrical parabolic solar collectors (CPC), which were improved through incorporation of reflective mirrors, thus increasing their efficiency. Similarly added a light monitoring system, based on photosensors, so we can maximize every sunbeam. As a first approximation it was assumed that the solar collector works as a perfect black body. Then we propose a more realistic model, where it takes into account the heat losses, dissipation of the solar rays, the optical properties of materials used, etc. In the experimental part, this system was assembled, and was compared with traditional systems of CPC. The principal result was a marked improvement in the temperature for the receiver tube in a shorter time of exposure. Also, we show applications of this type of collectors, ranging from domestic heating systems, improving production anaerobic fermentation, to thermal power plants for generating electricity. In the end, we show proposals for the improvement of this system according to theoretical investigation.

Keywords: cylindrical parabolic collector, light up, solar energy, reflective mirrors
\end{abstract}




\section{INTRODUCCIÓN}

Dos factores definieron que el uso de las energías renovables sea un campo muy activo el día de hoy:

- El gran daño que está causando el uso de combustibles fósiles al medio ambiente, y

- La escasez de este tipo de combustibles prevista para los siguientes años.

Por eso se necesitan propuestas diversas respecto a cómo se deben usar dichas energías renovables. Entre las fuentes clásicamente conocidas, como la energía solar, energía eólica, energía hidráulica, energía geotérmica, etc., centraremos nuestra atención en la primera porque el Sol es, quizá, la mayor posible fuente de energía, y veamos por qué.

Desde el punto de vista energético, la masa solar que por segundo se irradia al espacio en forma de partículas de alta energía y de radiación electromagnética es aproximadamente de 5,6×10 ${ }^{35}$ $\mathrm{GeV}$, y de ella la Tierra recibe en el exterior de su atmósfera un total de $1,73 \times 10^{14} \mathrm{~kW}$, es decir 1,353 $\mathrm{kW} / \mathrm{m}^{2}$ [1], la cual se conoce como constante solar y cuyo valor fluctúa en un $\pm 3 \%$ debido a la variación periódica de la distancia entre la Tierra y el Sol.

De toda la energía mencionada, el $30 \%$ es reflejado por la atmósfera al espacio exterior, el $23 \%$ se utiliza en la evaporación de los océanos, y el $47 \%$ se utiliza para calentar la atmósfera. Y una minúscula parte (menor al 1\%) se utiliza en los procesos de formaciones de vientos y fotosíntesis.

Del $47 \%$ de energía solar mencionada que se utiliza para calentar la atmósfera, solo el 31\% logra hacer contacto directo con la superficie terrestre, porque el otro $69 \%$ se pierde en el trayecto (ya sea por absorción de la tropósfera, reflejo de las nubes, el polvo, la humedad, etc.).

Estos valores mencionados son promedios sobre todo nuestro planeta. Esto se podría traducir en que, bajo condiciones óptimas, la radiación solar sobre la superficie terrestre podría llegar a $1 \mathrm{Kw} / \mathrm{m}^{2}$ (un valor muy ideal). Este valor no diverge mucho de la radiación solar recibida en determinadas regiones en nuestro país, lo cual lo hace uno de los más privilegiados en ese aspecto.
Como se vio, la presencia de la atmósfera reduce notablemente la radiación solar incidente sobre la superficie de la tierra, puesto que la atmósfera, en función a sus constituyentes gaseosos y aerosoles presentes en ella, tiene la capacidad de absorber, dispersar y reflejar la radiación solar. La cantidad de radiación que llega a la superficie terrestre es mayor si menor es la concentración de ozono, vapor de agua, dióxido de carbono y aerosoles en la atmósfera. La concentración y cantidad de los tres últimos disminuye con la altitud; por esta razón en el Perú, debido a su orografía, la radiación solar incidente aumenta considerablemente en la Sierra peruana. Esta región se encuentra principalmente entre los 2000 y 3000 metros de altitud y ocupa un 30\% del territorio nacional. Por tanto se puede notar que nuestro país tiene un potencial muy grande en cuanto a uso de este recurso.

El problema sigue siendo el mismo: las tecnologías aplicables dentro del mercado tienen costes muy elevados en comparación con el ingreso del campesino normal que habita en la sierra. Por ejemplo, si hablamos de paneles solares para generación de energía eléctrica, uno que solo genere 100 watts tiene un costo aproximado de 400 dólares (US\$). ¿Se le puede pedir a un campesino que gaste 400 dólares (omitiendo los gastos de instalación, mantenimiento y sistemas de regulación que un panel de por sí exige) para comprar un panel solar para que prenda un par de fluorescentes cuando él puede ir, cortar un árbol, y prender fuego sin gastar un solo dólar?

Este costo se debe a la poca eficiencia (15\%) que poseen estos aparatos. Aunque actualmente existen alternativas para poder obtener una mayor energía de un panel solar, un ejemplo claro son los paraboloides de revolución que se están utilizando en Australia para concentrar una mayor cantidad de energía solar sobre un panel solar.

En el presente trabajo, se presentará una alternativa para aprovechar la energía solar en procesos de calentamiento de agua a través de un sistema de colección solar parabólico automatizado. Esta alternativa es un poco más aplicable y rentable para nuestra población, debido a nuestro alto potencial en recursos solares y a que no necesita de especialistas en el tema, solo un poco de perseverancia. 


\section{Colectores solares cilíndricos parabólicos}

El esquema de este colector se puede ver en la figura 1. Este sistema aprovecha la propiedad de las parábolas: todo rayo que choque sobre la parábola será reflejado en dirección a un punto llamado foco. Es decir, que si se crea alguna superficie reflectora en forma de parábola, y se coloca un tubo receptor en ubicación exacta del foco, entonces este tubo recibirá todos los rayos solares de la superficie reflectora, logrando así una alta concentración de rayos solares sobre una pequeña región. Este tipo de colectores llegan hasta los $400{ }^{\circ} \mathrm{C}$.

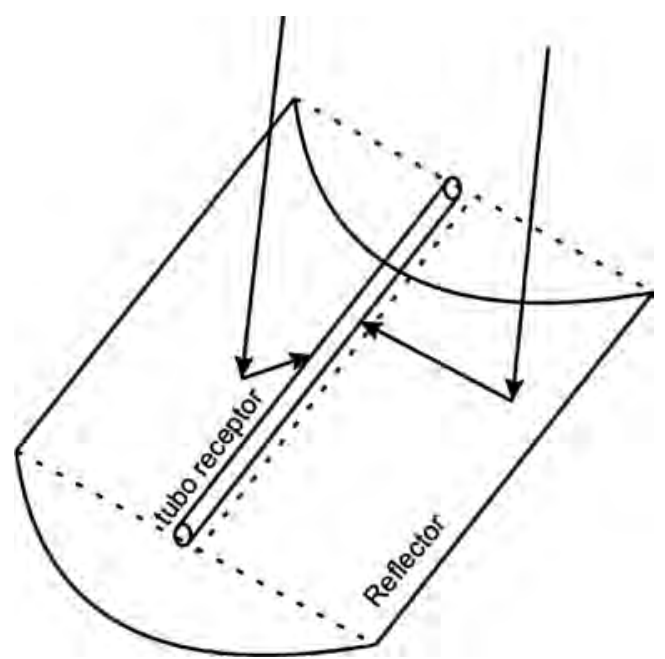

Figura 1: Esquema de un colector solar parabólico.

\section{Modelo teórico del colector solar parabólico}

Para empezar se debe recordar que la parábola se rige bajo la ecuación (asumiendo que comienza en el origen de coordenadas) $y^{2}=4 f x$, en donde $f$ es la distancia focal (fig. 2).

Llevando esa expresión a coordenadas polares, tenemos que:

$$
\rho=\frac{f}{\cos ^{2} \frac{\varphi}{2}}
$$

De acuerdo a la figura 2 , se puede llegar a la siguiente relación:

$$
h=2 f \operatorname{tg} \frac{\varphi}{2},
$$

recordando que el diferencial de longitud de arco de una parábola está dado por $d s^{2}=d \mathrm{r}^{2}+\mathrm{r}^{2} d \mathrm{j}^{2}$. Suponiendo que la parábola tenga una profundidad L, entonces el área total de la parábola será:

$$
A_{\Phi}=2 L f\left(\frac{\sin \left(\frac{\Phi}{2}\right)}{\cos ^{2}\left(\frac{\Phi}{2}\right)}+\ln \left|\operatorname{tg}\left(\frac{\pi}{4}+\frac{\Phi}{4}\right)\right|\right)
$$

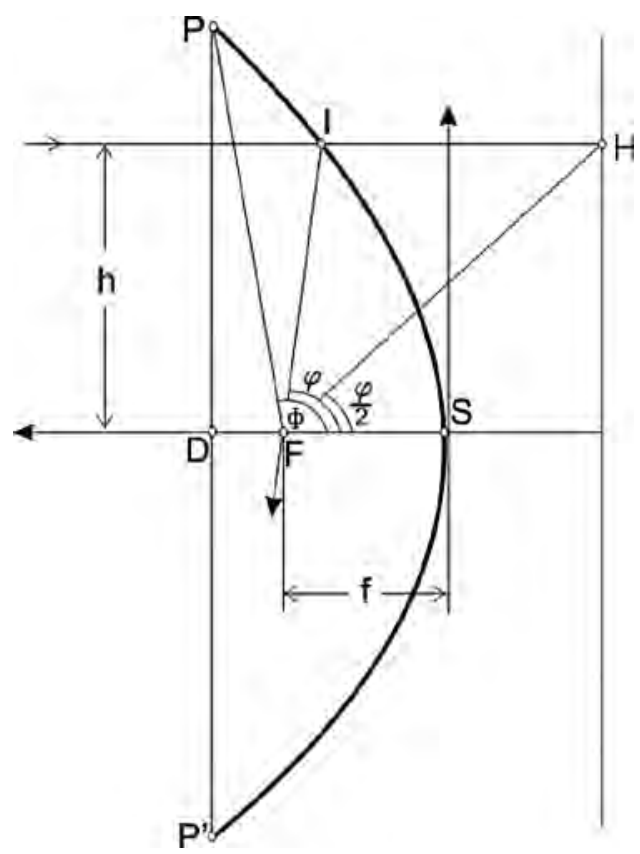

Figura 2: Esquema de una parábola.

Para futuros cálculos, resulta necesario definir el área total de la abertura de la parábola, la cual será importante porque la radiación solar se define sobre un área perpendicular al rayo solar (a donde llegará), que para este caso será el área de la abertura:

$$
A_{a}=4 L f \operatorname{tg} \frac{\Phi}{2}
$$

Es importante recalcar que de las ecuaciones 3 y 4 se puede notar que para ángulos grandes el aumento en la superficie del colector no genera un aumento proporcional en el área de la abertura (gráfico 1). Este detalle es el responsable de que la mayoría de colectores solares modernos no posean ángulos mayores a $60^{\circ}$ para no desperdiciar material en vano, ya que si aumentásemos el ángulo también se tendría que incluir un material extra, lo cual no se compensaría con la radiación extra obtenida.

Tomando en cuenta que el diámetro del sol es de $1,4 \times 10^{6} \mathrm{~m}$ y la distancia entre el sol y la tierra es de $1,5 \times 10^{8} \mathrm{~m}$, tenemos que el ángulo de abertura de los rayos solares que llegan a la tierra es de 32 minutos. A esta abertura se la denotará por $\xi$ (fig. 3). Por lo tanto, dentro del análisis es necesario asumir que el rayo que llega sobre un punto en la 
superficie reflectora no es solo una línea, sino que es considerada como un conjunto de rayos que se concentran sobre un punto en el colector.

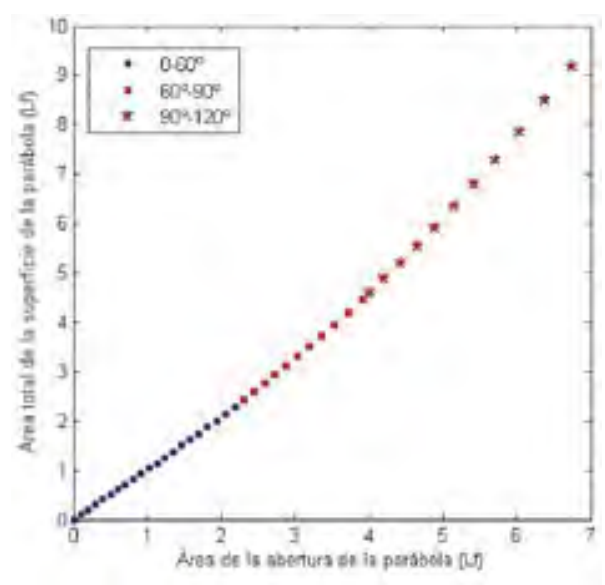

Gráfico 1: Relación entre las áreas totales y la abertura de la parábola.

Si se considera un haz de rayos luminosos de abertura $\varepsilon$ incidente en el punto I de la parábola, el haz reflejado corta al plano normal en el eje de la parábola básica que define el perfil del cilindro, según el eje mayor $\mathrm{AB}$. Como aproximación se puede asumir que el foco $\mathrm{F}$ de la parábola que pasa por el punto I es el centro de la elipse (fig. 3).

A diferencia del caso anterior, si ahora se considera un haz incidente de ángulo $\xi$ cuyo eje pasa por el vértice de la parábola I' que coincide con el haz reflejado, la imagen sobre el plano focal será un círculo de Gauss. Ahora se puede notar que si el punto de incidencia I' sobre el reflector se mueve a lo largo de la línea (I'I"), el círculo de Gauss se traslada a lo largo de un rectángulo de longitud L y anchura $f \xi$, limitado por dos semicírculos de radio $0,5 f \xi$, siendo $f$ la distancia focal de la parábola.

En algunas fuentes bibliográficas [2] se utiliza el análisis de la elipse de Gauss que se forma sobre el plano normal al plano de simetría para determinar los factores de concentración de este tipo de colectores. Pero si asumimos que el tubo receptor es cilíndrico y su centro se encuentra justo en el foco, entonces el cálculo se reduce a determinar el diámetro mínimo que debería tener el tubo receptor. Para ello, se extrae (fig. 3) el radio mínimo para que todos los rayos solares sean aprovechados por el receptor:

$$
\min _{\min }=\frac{f \xi}{\cos ^{2}\left(\frac{\Phi}{2}\right)}
$$

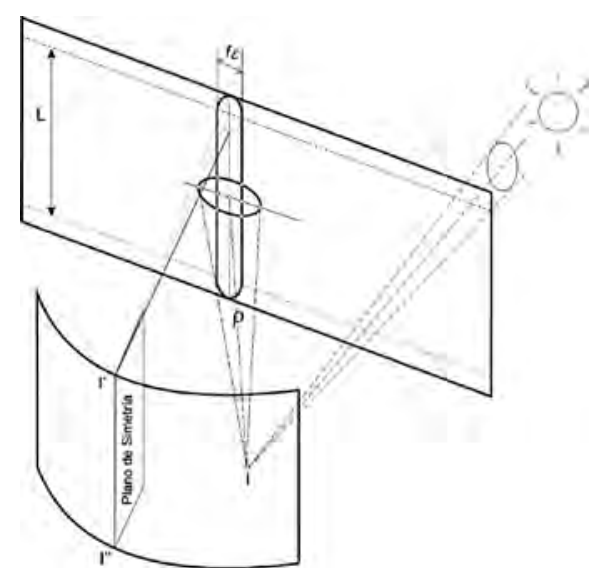

Figura 3: Esquema del ángulo de incidencia y reflexión.

Suponiendo que nuestro foco se encuentre a dos metros de nuestra superficie reflectante, y con una abertura de $\Phi=90^{\circ}$, entonces este radio mínimo será de $1 \mathrm{~cm}$, lo cual es fácilmente alcanzable. Pero en el presente trabajo, al utilizar espejos reflectantes, se utilizaron láminas de espejos de una longitud $L$ y un ancho $d$ (fig. 4). En la figura 4 se puede observar que los espejos fueron colocados de manera tal que formen una parábola; luego, mientras más alejados estén los espejos del foco, menor es la separación entre los rayos del inicio y fin del espejo respectivo. Así pues, no resulta difícil obtener que el radio mínimo necesario para aprovechar toda la radiación solar reflejada debe ser de:

$$
r_{\min }=\frac{f \xi}{\cos ^{2}\left(\frac{\Phi}{2}\right)}+\frac{d}{2}
$$

a)

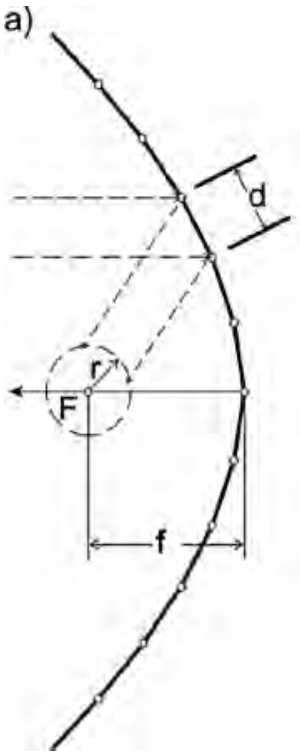

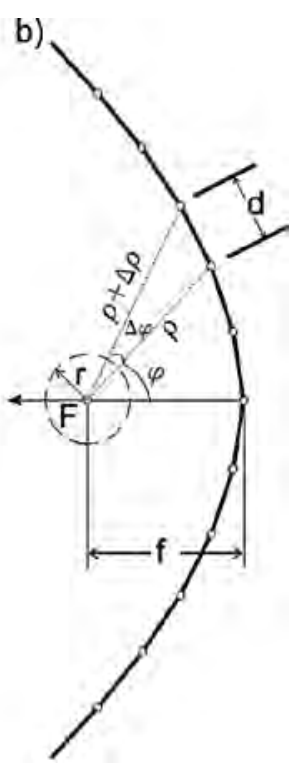

Figura 4: Esquema del prototipo real basado en láminas de espejos reflectantes. 


\section{Modelo del cuerpo negro}

Como primera aproximación, se podría tratar al tubo receptor como un cuerpo negro; esto es, asumimos que no existen pérdidas de ningún tipo (ya sea por radiación, conducción y convección).

Para ello, se debe tomar en cuenta que la superficie reflectante concentrará los rayos solares que caigan sobre el área de la abertura de dicha parábola en el área que ocupe el tubo receptor. Esto causará que la radiación incidente por unidad de área sobre el tubo colector sea mayor que la radiación recibida por la superficie terrestre. Al cociente de estas áreas se denomina factor de concentración, el cual matemáticamente se define como:

$$
C=\frac{A_{\text {abertura }}}{A_{\text {receptor }}}=\frac{2 f \operatorname{tg}\left(\frac{\Phi}{2}\right)}{r_{\text {receptor }}}
$$

En donde se utilizó la ecuación (4), y el área transversal del tubo receptor es $2 r L$. La gráfica 2 muestra el comportamiento del factor de concentración en función del área total de la parábola, para un radio del receptor igual a una centésima de la distancia focal.

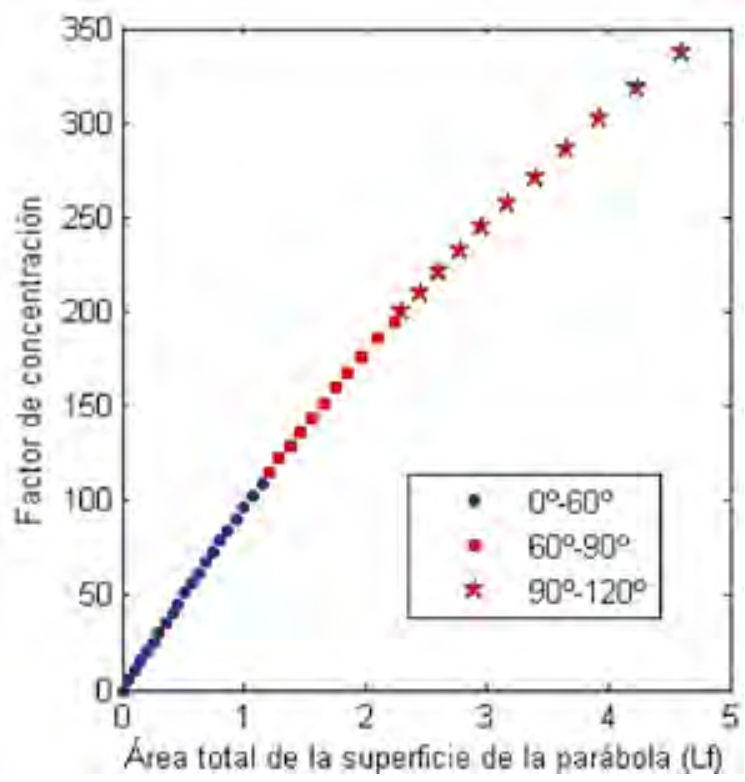

Gráfico 2: Relación entre el factor de concentración y el área total de la superficie de parábola.

Para poder plantear nuestro modelo del cuerpo negro, se debe recurrir a la ecuación de Stefan Boltzmann, incluyendo el factor de concentración para lo cual:

$$
\mathrm{IC}=\sigma \mathrm{T}^{4}
$$

donde $\mathrm{C}$ es el factor de concentración, $\mathrm{T}$ la temperatura, I la energía incidente sobre el reflector, $\sigma$ la constante de Boltzmann, cuyo valor es $5,6704 \times 10^{-8} \mathrm{~h}$.

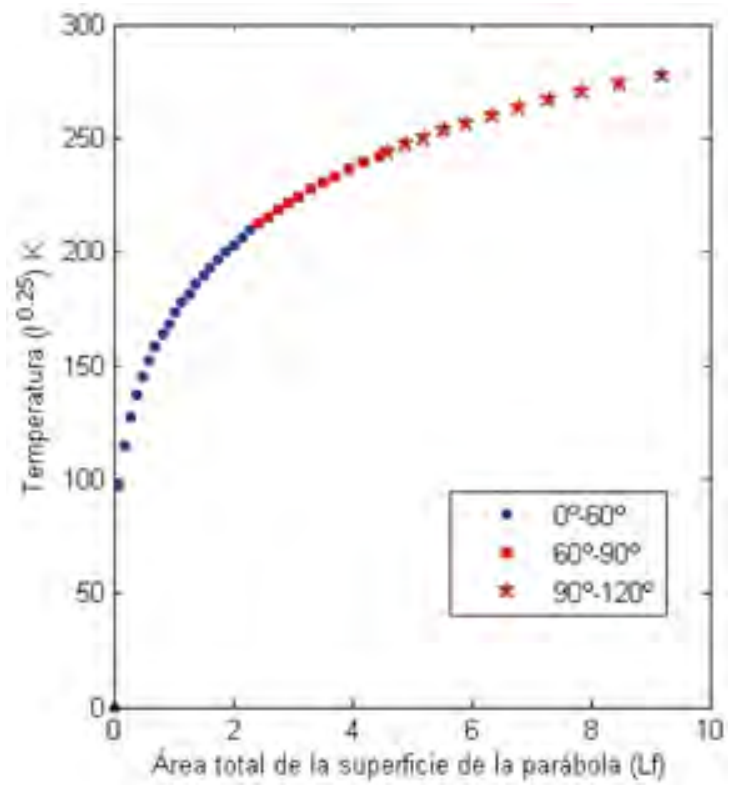

Gráfico 3: Relación entre el factor de concentración el área total de la parábola.

\section{Modelo más realista}

En el modelo anterior se asumió que toda la radiación sería absorbida por el tubo receptor y sería automáticamente transformada en calor, lo cual se manifiesta en el aumento de temperatura del mismo.

Se debe recordar que la intención final de este colector no es calentar el tubo receptor propiamente dicho, sino el agua que circulará dentro de ella. Para ello se tiene que la radiación solar que afecte al tubo receptor generará un calor útil que será el responsable de que el agua llegue a calentarse. Dentro de tal proceso existen pérdidas de calor por conexión, conducción y radiación (fig. 5).

Utilizando las ecuaciones de la termodinámica, se puede llegar a la siguiente expresión:

$$
\dot{q}_{\text {util }}=\dot{m}_{\text {agua }} \cdot c \cdot\left(T_{\text {salida }}-T_{\text {ingreso }}\right)=\alpha \tau I A-\dot{Q}_{\text {perdidas }} \text {, }
$$

en donde $\alpha$ es la absorbancia y $\tau$ es la transmitancia del tubo receptor; $\mathrm{T}$ salida y $\mathrm{T}$ ingreso son las 
temperaturas de salida e ingreso del agua; I es la radiación solar; A es área de la abertura de la parábola y $\mathrm{C}$ es el calor específico del agua. El calor perdido por convexión, radiación y conducción están dados por:

$$
\begin{aligned}
& \dot{Q}_{\text {perdidas }}(h)=h \cdot A_{r} \cdot\left(T_{\text {receptor }}-T_{\text {ambiente }}\right) \\
& \dot{Q}_{\text {perdidas }}(\varepsilon)=\varepsilon \cdot \sigma \cdot A_{r} \cdot\left(T_{\text {recepor }}^{4}-T_{\text {ambieme }}^{4}\right) \\
& \dot{Q}_{\text {perdidas }}(k)=k \cdot \Delta x \cdot A_{r} \cdot\left(T_{\text {receptor }}-T_{\text {ambiente }}\right)
\end{aligned}
$$

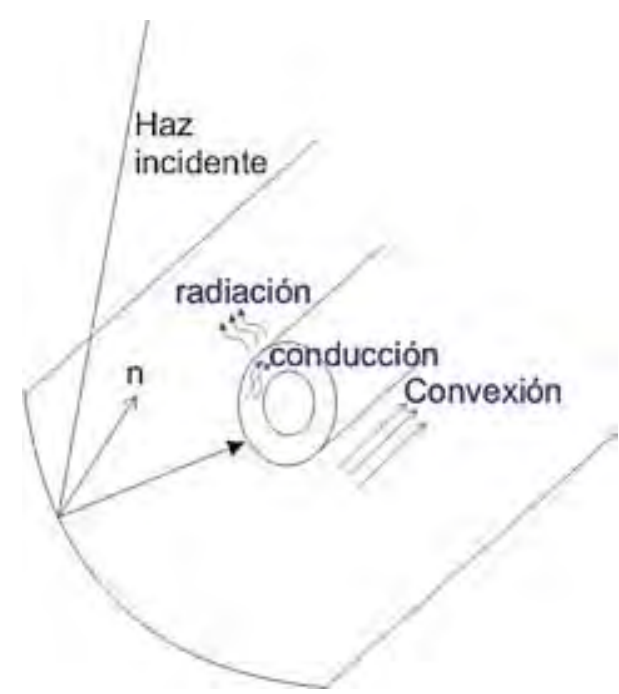

Figura 5: Esquema de las pérdidas de calor del sistema.

En el análisis de este tipo de colectores, es necesario el uso del ángulo $\theta[3]$ (se forma entre el vector radiación del sol y el vector perpendicular a la superficie normal al plano de simetría de la parábola). Para lo cual tenemos que la energía incidente por unidad de superficie sobre el concentrador reflector es:

$$
I=I_{0} \cos \theta
$$

El ángulo $\theta$ mencionado en la ecuación anterior tendrá determinados valores dependiendo del tipo de sistema de seguimiento que se implante en el colector.

En puntos diferentes al ecuador, un colector solar que pretenda seguir el movimiento completo del sol, necesitaría dos ejes de giros perpendiculares entre sí. Para ello existen dos propuestas: la primera es que se genere un sistema de rotación de este a oeste y otro de norte a sur, y la segunda que se gire alrededor de un eje perpendicular al de la tierra y otro inclinado. Esto causaría que el ángulo $\theta$ sea igual a cero durante todo el día, lo cual llevaría a que la intensidad captada por esos sistemas sea el máximo posible, de acuerdo a la ecuación 9. Como en el caso de este estudio se trabajó con un CCP automatizado, entonces en la ecuación 9 el ángulo será siempre cero grados.

Si se pretende encontrar alguna cota para el caso de transferencia de calor ideal, asumimos que en el sistema no existen pérdidas de calor. El gráfico 4 muestra el comportamiento ideal.

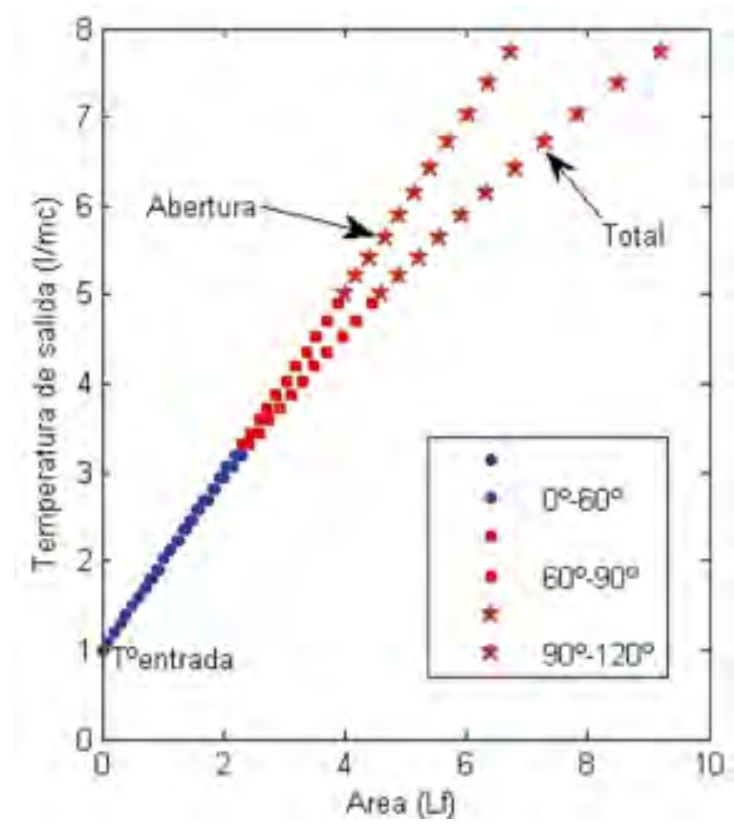

Gráfico 4. Relación entre la temperatura de salida del fluido y las áreas totales y de la abertura del CCP.

Los diferentes coeficientes que causan las pérdidas de calor son determinados experimentalmente.

\section{MÉTODO EXPERIMENTAL}

En el desarrollo experimental, se adecuaron a modo de comparación el sistema de reflexión y un sistema de seguimiento solar automatizado, donde se pudo observar diferencias con un CCP construido con materiales reflectantes, como el acero inoxidable, y un sistema de seguimiento solar asistido. Los cálculos corroboran el mejor funcionamiento del sistema adecuado para este trabajo. Asimismo, proponemos que el sistema sea asistido por un módulo solar (celda fotovoltaica), el cual pueda dotarnos de la fuente eléctrica requerida para accionar el motor, pues la fuente de energía eléctrica actualmente utilizada es la convencional.

La figura 6 muestra la imagen general del sistema de CCP dotado de sistema de seguidor solar automatizado. 


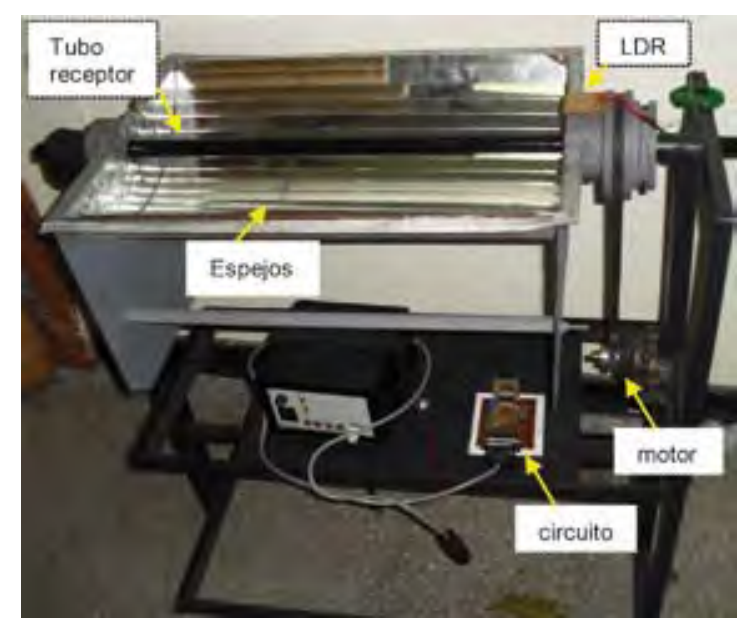

Figura 6: CCP automatizado con espejos reflectantes construido.

La tabla 1 presenta un cuadro comparativo con dos tipos de CCP.

Tabla 1: Cuadro comparativo de los colectores parabólicos diseñados por el GIFUNT y el presentado en este trabajo.

\begin{tabular}{|c|c|c|}
\hline & $\begin{array}{l}\text { Colector cilíndrico } \\
\text { parabólico } \\
\text { (GIFUNT) }\end{array}$ & $\begin{array}{l}\text { Colector } \\
\text { cilíndrico } \\
\text { parabólico } \\
\text { (UPN) }\end{array}$ \\
\hline \multicolumn{3}{|c|}{ Características del equipo CCP: } \\
\hline $\begin{array}{l}\text { Longitud del foco } \\
\text { parabólico }\end{array}$ & $65 \mathrm{~cm}$ & $65 \mathrm{~cm}$ \\
\hline Diámetro focal & $1 / 2 “$ & $1 / 2$ “ \\
\hline $\begin{array}{c}\text { Material del foco } \\
\text { parabólico }\end{array}$ & Cobre & Cobre \\
\hline $\begin{array}{c}\text { Largo parabólico } \\
\text { (horizontal) }\end{array}$ & $65 \mathrm{~cm}$ & $65 \mathrm{~cm}$ \\
\hline Material reflejante & Acero inoxidable & Espejo \\
\hline $\begin{array}{c}\text { Ángulo de giro } \\
\text { parabólico }\end{array}$ & $180^{\circ}$ & $180^{\circ}$ \\
\hline $\begin{array}{l}\text { Intervalo de tiempo } \\
\text { estacionario (de pase } \\
\text { de agua por el foco } \\
\text { geométrico) }^{(*)}\end{array}$ & $6-7$ minutos & $4-5$ minutos \\
\hline \multicolumn{3}{|c|}{ Resultados obtenidos: } \\
\hline $\mathbf{T}^{\circ}$ Ingreso del agua & $20,5^{\circ} \mathrm{C}$ & $20,0^{\circ} \mathrm{C}$ \\
\hline$T^{\circ}$ Salida del agua & $73^{\circ} \mathrm{C}$ & $77^{\circ} \mathrm{C}$ \\
\hline \multicolumn{3}{|c|}{ Datos adicionales (características del día de toma de datos): } \\
\hline $\mathrm{T}^{\circ}$ Ambiente & $20.5^{\circ} \mathrm{C}$ & $20{ }^{\circ} \mathrm{C}$ \\
\hline $\begin{array}{l}\text { Intensidadderadiación } \\
\text { promedio }\end{array}$ & $\begin{array}{c}700 \mathrm{~W} / \mathrm{m} 2-750 \\
\mathrm{w} / \mathrm{m} 2\end{array}$ & $\begin{array}{c}700 \mathrm{~W} / \mathrm{m} 2- \\
750 \mathrm{~W} / \mathrm{m} 2\end{array}$ \\
\hline OTROS & $\begin{array}{l}\text { Sistema de } \\
\text { seguimiento solar } \\
\text { asistido }\end{array}$ & $\begin{array}{c}\text { Sistema de } \\
\text { seguimiento } \\
\text { solar } \\
\text { automatizado } \\
\end{array}$ \\
\hline
\end{tabular}

(*) El intervalo de pase de agua por el foco geométrico de parábola fue asistido mediante una válvula de goteo continuo adherido a la salida del tanque principal de agua y graduado en $4.86 \mathrm{ml} / \mathrm{min}$ (aprox. $5 \mathrm{ml} / \mathrm{min}$.) a un paso de longitud de $65 \mathrm{~cm}$. Se comparó con el diseño creado por el Grupo de Investigación de Física de la Universidad Nacional de Trujillo (GIFUNT)
Como se mencionó antes, para sustentar este trabajo se desarrollaron dos prototipos con las mismas características de construcción (tabla 1), salvo respecto a dos elementos: el material reflectante y el sistema de seguimiento solar. Cabe mencionar que los resultados obtenidos de temperatura fueron tomados en época de verano (mes de febrero).

\section{RESULTADOS Y DISCUSIÓN}

En la actualidad existe gran demanda de sistemas de colectores cilíndricos parabólicos de gran envergadura en centrales térmicas solares, todas ellas accionadas con sistema computarizado de seguimiento solar. Ante eso, en este trabajo se propuso un sistema de seguimiento solar por fotosensores, además del análisis comparativo de variaciones térmicas de dos sistemas de CCP con medio reflectante diferentes (espejo vs. acero inoxidable). El más eficiente fue el CCP con espejo, quizás debido al sistema de seguidor solar que se le adhirió, mediante la construcción de una montura de seguimiento de azimut de tipo ecuatorial capaz de seguir al sol de manera autónoma y con un gasto energético que puede ser fácilmente suministrado por un panel fotovoltaico. Esto deja abierta la posibilidad de la instalación de un módulo fotovoltaico que recargue constantemente las baterías que alimentan al motor.

Las aplicaciones de los colectores parabólicos se han generalizado, desarrollándose más los diseños de centrales solares para la generación de energía eléctrica, seguido de aplicaciones de viviendas con diseño bioclimático, así mismo para el mejoramiento de deshidratadores solares como sistemas de precalentamiento de destiladores solares, y el aumento de la temperatura en cámaras de generación de biogás, entre otros [4].

La aplicación más inmediata de este tipo de sistemas de colección solar en la sierra peruana es la posible implementación de un sistema de calefacción para viviendas. La conocida oleada de frío que aqueja a esas regiones y el tremendo potencial solar debido a la altitud a la que se encuentran, hace que un sistema de colección como un posible panel fotovoltaico podría tener muy buenos resultados bajo esas condiciones. Con este sistema no habría la preocupación de que el panel se cubra con 
alguna capa de hielo (tal como está pasando en la actualidad), pues el mismo colector suministraría el calor al panel, evitando cualquier solidificación del agua del ambiente.

Del análisis teórico queda claro, además, que si manejamos adecuadamente los coeficientes que causan las pérdidas de energía en el sistema, podríamos aumentar aún más la eficiencia para la misma. Entre estas mejoras está la utilización de espejos de primera superficie que permitiría obtener hasta un $5 \%$ más de energía recibida por el colector. También se podría recubrir el tubo receptor con vidrio, tal que genere un efecto invernadero y aumente así la temperatura total del tubo receptor (aquí se debe tener cuidado, pues la calentura podría romper el espejo; por tanto se requiere otro estudio para probar la factibilidad de esta propuesta).

\section{CONCLUSIONES}

- Se logró construir con éxito el CCP automatizado con espejos reflectantes.

- Las temperaturas alcanzadas por este tipo de CCP son ligeramente mayores (en un 6\%) que las obtenidas por el CCP basado en acero galvanizado.

- El tiempo de espera para calentar determinados volúmenes de agua es menor (en un 30\%) en el CCP basado en espejos reflectantes que en el de acero galvanizado.

- Se logró construir un modelo físico-matemático del CCP que nos permite establecer cotas máximas (teóricas) esperadas para estos colectores.
- Este sistema podría haberse mejorado si se hubiese colocado alguna cubierta de vidrio al tubo receptor, tal que por efecto invernadero, las temperaturas y los tiempos obtenidos hubiesen sido mejores.

- Queda abierta la posibilidad del uso de espejos de primera superficie para la mejora del coeficiente de reflexión de los espejos de la superficie del CCP.

- El sistema podría ser autosostenible si se le implementa algún sistema de celdas solares tal que los motores puedan funcionar con la energía generada por tales celdas.

\section{AGRADECIMIENTOS}

Un agradecimiento especial al Mg. Francisco Rodas Díaz, por su constante apoyo durante el desarrollo del presente trabajo.

\section{REFERENCIAS}

[1] Solar Constant, consultada el 6 de junio del 2010 en: http://www.pmodwrc.ch/pmod.php?topic=tsi/ composite/SolarConstant.

[2] P. Fernández Diez, Procesos Termosolares en Baja, Media y Alta Temperatura, Departamento de Energía Eléctrica y Energética, Universidad de Cantabria, 2007.

[3] C. Polo Bravo y E. Sacari Sacari, "Evaluación de un Concentrador Cilíndrico Parábolico", XVI Simposio Peruano de Energía Solar, 2009.

[4] J. Quinteros Grijalva, Estudio teórico y experimental de colector solar parabólico para generación de energía eléctrica, tesis, Universidad de Chile, 2008.

E-mail: pia@upnorte.edu.pe davidasmat88@hotmail.com 